\title{
Comparison of pre-emptive and reactive strategies to control an incursion of bluetongue virus serotype 1 to Great Britain by vaccination
}

\author{
T. SUMNER ${ }^{1 *}$, L. BURGIN ${ }^{2}$, J. GLOSTER ${ }^{2}$ AND S. GUBBINS ${ }^{1}$ \\ ${ }^{1}$ Institute for Animal Health, Pirbright Laboratory, Pirbright, Surrey, UK \\ ${ }^{2}$ The Met Office, Exeter, UK
}

Received 21 September 2011; Final revision 7 February 2012; Accepted 8 March 2012; first published online 4 April 2012

\section{SUMMARY}

Bluetongue (BT) is a disease of ruminants caused by bluetongue virus (BTV), which is spread between its hosts by Culicoides midges. Vaccination is the most effective way to protect susceptible animals against BTV and was used reactively to control the recent northern European outbreak. To assess the consequences of using vaccination pre-emptively we used a stochastic, spatially explicit model to compare reactive and pre-emptive vaccination strategies against an incursion of BTV serotype 1 (BTV-1) into Great Britain. Both pre-emptive and reactive vaccination significantly reduced the number of affected farms and limited host morbidity and mortality. In addition, vaccinating prior to the introduction of disease reduced the probability of an outbreak occurring. Of the strategies simulated, widespread reactive vaccination resulted in the lowest levels of morbidity. The predicted effects of vaccination were found to be sensitive to vaccine efficacy but not to the choice of transmission kernel.

Key words: Bluetongue, control, epidemiology, mathematical modelling.

\section{INTRODUCTION}

Bluetongue (BT) is a disease of ruminants caused by bluetongue virus (BTV), an arthropod-borne virus which is spread between its hosts by the bites of Culicoides midges. Once regarded as a tropical and subtropical disease, the range of BT has increased considerably in recent years extending into northern Europe [1-3]. BTV serotype 8 (BTV-8) was first reported in The Netherlands in 2006 and throughout that year spread to Germany, Belgium, France and Luxembourg [2, 4]. In 2007 BTV-8 re-emerged in the previously affected countries and spread into new areas including Great Britain (GB) [5]. Inactivated

* Author for correspondence: Dr T. Sumner, Institute for Animal Health, Pirbright Laboratory, Ash Road, Pirbright, Surrey GU24 0NF, UK.

(Email: t.sumner@iah.ac.uk) vaccines against BTV-8 became commercially available in early 2008 and vaccination programmes were initiated in most affected countries. The success of vaccination varied from country to country but where uptake was high, vaccination appears to have been largely effective in controlling transmission of BTV-8 [3]. In particular, GB, which began a voluntary vaccination programme in May 2008, has reported no new cases of BTV-8 infection since 2007. While the precise reason for the lack of subsequent BTV cases in GB is unclear, conditions for disease introduction (circulation of disease on the continent and winds suitable for the transport of infected midges to GB) and transmission (appropriate temperatures) were favourable during 2008 [6]. It is therefore reasonable to assume that vaccination played a major role in preventing the re-occurrence of BTV-8 in GB. 
Vaccination programmes against BTV-8 were all implemented reactively, i.e. once an outbreak had occurred. This was primarily a consequence of the unexpected nature of the original incursion [3, 7] and the lack of a suitable vaccine [3]. It is, however, possible to conceive of using vaccine in a pre-emptive manner (i.e. prior to an outbreak), where there is the risk of an incursion of BTV (e.g. the presence of BTV in a neighbouring region) and a vaccine is available. Before implementing such a strategy it is important to assess the consequences of pre-emptive vaccination for an incursion of BTV and to compare this with alternative reactive strategies. In this paper we present such a comparison of pre-emptive and reactive vaccination against an incursion of BTV-1. This serotype had spread northwards in Europe through Spain and Portugal during 2007 and 2008, and by 2008 was circulating in northern France $[3,8]$ and, hence, posed a risk to UK livestock via windborne dispersal of infected vectors $[6,9,10]$.

To assess different vaccination strategies against BTV and their ability to control the spread of disease we used a stochastic spatially explicit model of BTV transmission within and between farms [11, 12] to evaluate different scenarios for the deployment of vaccine in the event of an introduction of BTV into GB. Specifically, we compared pre-emptive and reactive strategies in terms of their impact on: (i) the probability of an incursion resulting in an outbreak (i.e. secondary spread from the initial incursion); and (ii) outbreak size (both the number of holdings and spatial extent of spread). The impact of vaccination was assessed for different incursion dates and a range of levels of vaccine uptake by farmers. We also assessed the sensitivity of the conclusions to assumptions about the shape of the transmission kernel (i.e. the probability of transmission between farms) and vaccine efficacy.

\section{METHODS}

\section{Modelling framework}

Details of the model used to describe the transmission of BTV within and between farms can be found in Szmaragd et al. [11]. A summary of the model is provided below.

(i) Within-farm transmission was described by a stochastic model which includes two host species (cattle and sheep) and a single vector species. Parameter estimates, applicable to GB where possible, were obtained from the literature. Temperature-dependent functions were used for the reciprocal of the time interval between blood meals (related to the biting rate), the vector mortality rate and the duration of the extrinsic incubation period (EIP). The duration of viraemia in hosts was described by a gamma distribution with point estimates for the parameters. Other parameters were sampled from appropriate ranges for each farm.

(ii) Spread between farms was described by a stochastic, spatially explicit farm-level model with a daily time step in which the probability of transmission depends on the distance between farms and the host species composition of the farms. The distance dependence was described by a generic transmission kernel which includes both host movements and vector dispersal. Parameters for the transmission probability were estimated using data from the 2006 outbreak in northern Europe. Unless otherwise stated, the best-fit Gaussian kernel was used [11].

\section{Incursion of BTV-1 into GB}

The model was initialized by the introduction of infectious vectors to a single farm via the windborne dispersal of vectors from northern France. The risk of incursion was assessed using the UK Met Office's atmospheric dispersion model NAME (numerical atmospheric-dispersion modelling environment) [13]. This model has been adapted to incorporate the effects of meteorology on the flight of Culicoides midges [14] based on the results of field and laboratory studies carried out by the Institute for Animal Health (C. Sanders, personal communication). The model was used to calculate the number of occurrences of winds suitable for midge transport from four sites on the northern coast of France (Brittany, Lower Normandy, Upper Normandy, North Calais) to each county in southern England over the period May-October for the years 2006-2008. These sites were chosen as the most likely sources of windborne infected Culicoides due to their proximity to GB. While no data were available on the abundance of Culicoides at these sites, BT cases have been reported in the region indicating the presence of competent vectors. The results of the NAME analysis were used to quantify the relative frequency of potential introduction events to each county. The introduction site for the simulations was chosen by selecting a 
county at random based on these weightings and selecting a random farm within that county.

\section{Vaccination}

Vaccination was assumed to act by reducing the probability of transmission from vector to host $(b)$, by lowering the probability that an animal acquires infection, and from host to vector $(\beta)$ owing to reduced virus titres in infected, vaccinated animals. The probabilities decreased linearly over time from their baseline values $(b, \beta)$ at the time of vaccination to their minimum values $[b(1-\varepsilon), \beta(1-\varepsilon)$, where $\varepsilon$ is the vaccine efficacy] at full protection [12]. The vaccine was assumed to be $100 \%$ efficient unless stated otherwise. The time to full protection was assumed to be 14 days post-vaccination in sheep and 42 days in cattle based on information supplied by the vaccine manufacturers. The model assumes that there is no reduction in the duration of viraemia in infected vaccinated animals. The total number of vaccine doses used (one per sheep and two per bovine) is recorded but is assumed not to be limiting.

\section{Data}

The location and livestock populations (numbers of cattle and sheep) on each holding were obtained from the June agricultural survey data for 2006. Hourly temperature records for 2007 were extracted from the MIDAS database accessed from the British Atmospheric Data Centre (BADC) [15] for each of the 19 meteorological stations used in the model [11].

\section{Scenarios}

Thirteen vaccination scenarios were considered in order to investigate the impact of different strategies on the spread of BTV in GB (see Table 1). Scenario 1 represented a baseline in which no vaccine was used. Scenarios 2-7 described reactive vaccination strategies. Reactive vaccination was simulated in response to the detection of infected premises. A farm was identified as infected if an animal died due to disease or if overt clinical signs were observed in at least one animal [11]. Unvaccinated farms within a specified radius of an infected holding were vaccinated with probability given by the uptake level of reactive vaccine, and all cattle and sheep on the holding were assumed to be vaccinated. Farms were vaccinated in a random order, commencing 2 days after the detection
Table 1. Details of vaccination scenarios included in the analysis. Each scenario was simulated for incursions on 1 May, 1 July and 1 September

\begin{tabular}{lclll}
\hline \hline Scenario & $\begin{array}{l}\text { Radius } \\
(\mathrm{km})\end{array}$ & $\begin{array}{l}\text { Reactive } \\
\text { uptake } \\
(\%)\end{array}$ & VZ & $\begin{array}{l}\text { VZ } \\
\text { uptake } \\
(\%)\end{array}$ \\
\hline 1 & 0 & - & None & - \\
2 & 20 & 80 & None & - \\
3 & 20 & 95 & & \\
4 & 50 & 80 & & \\
5 & 50 & 95 & & \\
6 & 100 & 80 & & \\
7 & 100 & 95 & & 50 \\
8 & 20 & 95 & 1 & 50 \\
9 & & & 2 & 80 \\
10 & & & 1 & 80 \\
11 & & & 2 & 95 \\
12 & & & 1 & 95 \\
13 & & & 2 & \\
\hline \hline
\end{tabular}

VZ, Vaccination zone.

of the infected farm, to account for the time taken to confirm the diagnosis of BT, and at a constant number of farms per day, such that all farms were vaccinated within 21 days [12]. The number of farms vaccinated per day may vary depending on the number of farms in proximity to the infected farm. This is consistent with the fact that BT vaccination is carried out by farmers and, hence, the rate is not limited by the availability of specialist teams. Three radii $(20,50$, $100 \mathrm{~km})$ and two levels of uptake $(80 \%, 95 \%)$ were considered.

Scenarios 8-13 described pre-emptive vaccination strategies in which vaccine was used prior to the introduction of BTV-1 into GB. Vaccination was based on the declaration of a vaccination zone (VZ). Farms within the zone were vaccinated with probability given by the level of vaccine uptake. Vaccination commenced on 1 April and occurred at a constant number of farms per day such that all farms to be vaccinated were vaccinated within 21 days. Three levels of uptake $(50 \%, 80 \%, 95 \%)$ and two different zones (Fig. 1) were considered. The consideration of an additional lower level of uptake $(50 \%)$ for the preemptive scenarios reflects the possibility that farmers may be less likely to vaccinate when the perceived risk of disease is low. The first zone (VZ 1) was made up of the eight counties on the south coast of England regarded as at risk of an introduction of BTV-1. The second zone (VZ 2) consisted of the 25 counties south of a line extending from the mouth of the Severn in 
(a)

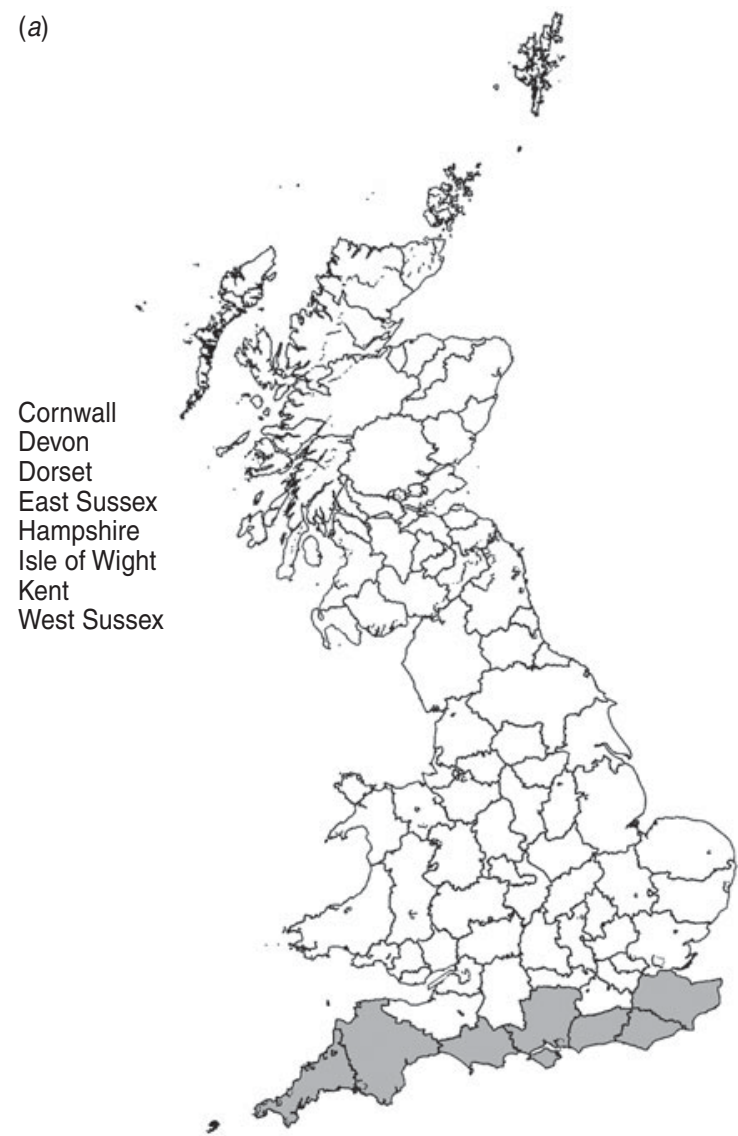

(b)

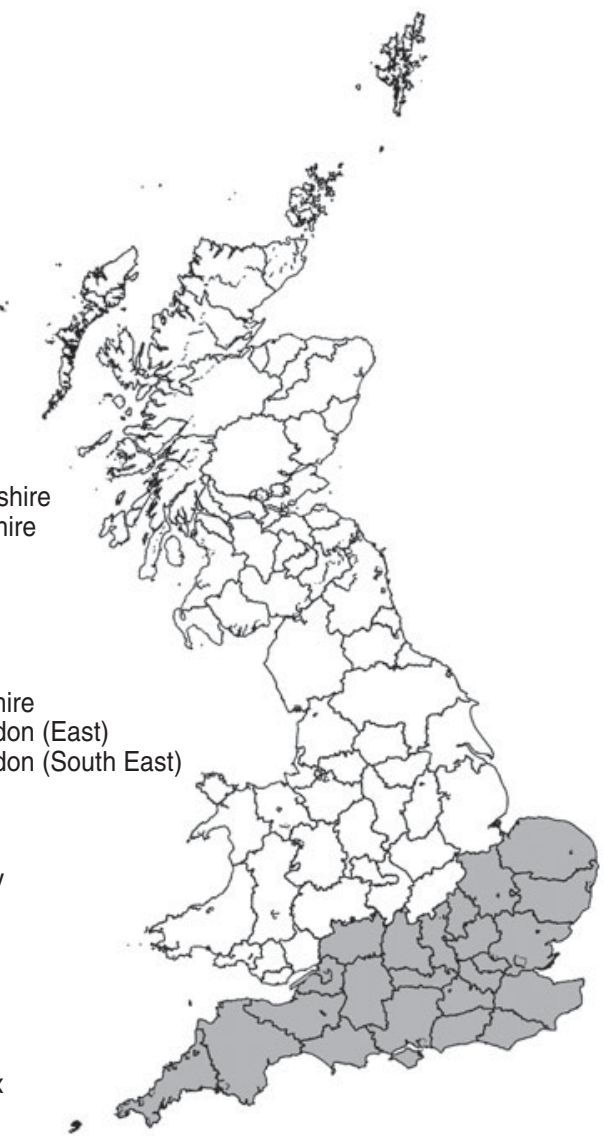

Fig. 1. Vaccination zones for bluetongue virus serotype 1 (BTV-1). Shaded areas indicate the counties (listed) within the zone. (a) Zone 1 based on the counties at risk of a windborne incursion of BTV-1. (b) Zone 2 which includes all counties south of a line extending from the mouth of the Severn in the west to the Wash in the east.

the west to the Wash in the east. In each of these scenarios additional reactive vaccination was simulated (as described above) in a $20-\mathrm{km}$ zone around clinically affected premises with $95 \%$ uptake.

For each scenario, simulations were run for three introduction dates (1 May, 1 July, 1 September) until 31 December. For each scenario sufficient replicates of the model were run to generate 30 outbreaks (defined as any spread from the initially infected farm). Those replicates where secondary spread did not occur were discarded. This approach ensures that sufficient outbreaks were simulated to produce robust results. The number of replicates is not specified in advance; rather it follows a negative binomial distribution.

\section{Sensitivity analysis}

Sensitivity analysis was conducted to assess the impact of vaccine efficacy $(\varepsilon)$ and the shape of the transmission kernel on the predicted impacts of vaccination. Vaccine efficacy was allowed to vary between $50 \%$ and $100 \%$ in $10 \%$ increments. Four kernels were used: Gaussian; exponential; fat-tailed (all with parameter estimates obtained using data on farms clinically affected by BTV-8 in northern Europe in 2006 [11]); and, the kernel estimated from the 2001 outbreak of foot-and-mouth disease (FMD) in GB $[16,17]$.

The following vaccination strategies were simulated for each kernel assuming $100 \%$ efficacy: (i) no vaccination; (ii) reactive vaccination uptake of $95 \%$ over a radius of 20,50 or $100 \mathrm{~km}$; (iii) $80 \%$ vaccine uptake in either VZ 1 or VZ 2 with reactive vaccination uptake of $95 \%$ over a radius of $20 \mathrm{~km}$. The following strategies were simulated for each value of efficacy using the Gaussian kernel: (i) reactive vaccination uptake of $95 \%$ over a radius of 20,50 or $100 \mathrm{~km}$; (ii) $80 \%$ vaccination in VZ 1 with reactive vaccination uptake of $95 \%$ over a radius of $20 \mathrm{~km}$. A total of 48 scenarios were considered, full details of which are provided in Table S1 of the Supplementary material. 
Table 2. Occurrences of wind events suitable for the transport of Culicoides midges from northern France to each county on the south coast of England

\begin{tabular}{llllllll}
\hline \hline & Cornwall & Devon & Dorset & Hampshire & Isle of Wight & Sussex & Kent \\
\hline May & $1 \cdot 3$ & $2 \cdot 3$ & 1 & $1 \cdot 7$ & 3 & $3 \cdot 3$ & $2 \cdot 7$ \\
June & $0 \cdot 7$ & $0 \cdot 7$ & 1 & $1 \cdot 7$ & $1 \cdot 3$ & 8 & $8 \cdot 7$ \\
July & $0 \cdot 7$ & $1 \cdot 7$ & 3 & 3 & $2 \cdot 3$ & $7 \cdot 3$ \\
August & 0 & $1 \cdot 3$ & $1 \cdot 7$ & $4 \cdot 3$ & $6 \cdot 7$ & $8 \cdot 3$ \\
September & $3 \cdot 7$ & 4 & 3 & $6 \cdot 7$ & $5 \cdot 7$ & $8 \cdot 3$ \\
October & $1 \cdot 7$ & $3 \cdot 7$ & 4 & 6 & 12 \\
\hline \hline
\end{tabular}

Counties are listed from west to east. Values represent the mean number of events per month from all sites in the years 2006-2008.

Table 3. Relative frequencies of winds that could carry infected midges from northern France to the counties on the south coast of England, estimated from the NAME analysis

\begin{tabular}{llllllllll}
\hline \hline & Cornwall & Devon & Dorset & Hampshire & Isle of Wight & W. Sussex & E. Sussex & Kent \\
\hline Relative frequency of incursion & 1 & 1 & 3 & 3 & 3 & 6 & 6 & 6 \\
\hline \hline
\end{tabular}

NAME, Numerical atmospheric-dispersion modelling environment.

East Sussex and West Sussex are represented as separate counties in the epidemiological model and are assumed to be at equal risk of an introduction of bluetongue virus.

All scenarios were simulated for incursions on 1 May and 1 July.

\section{Statistical analysis}

The probability of an incursion resulting in an outbreak was analysed using generalized linear models (GLM) with quasi-binomial errors (to account for overdispersion) and a logit link function. Outbreak sizes (number of affected farms) were compared using GLM with negative binomial errors and a log link function. Model construction proceeded by stepwise deletion of non-significant $(P<0 \cdot 05)$ terms starting from an initial model including date of incursion and choice of $\mathrm{VZ}$ as categorical predictors, and level of vaccine uptake in the VZ, radius for reactive vaccination and uptake of reactive vaccine as continuous predictors, together with pairwise interactions between all factors. The analysis was performed in the statistical programming language $\mathrm{R}$ [18].

\section{RESULTS}

\section{Incursion of BTV-1 into GB}

All counties on the south coast of England were found to be at risk, with the eastern-most counties (Kent and Sussex) at greatest risk and Cornwall at the lowest risk (Table 2). Based on these results the relative frequency of potential introduction events for each county was estimated (Table 3), and these were used when simulating incursions.

\section{Probability of an outbreak}

The proportion of incursions which resulted in an outbreak in the absence of vaccination and for each pre-emptive vaccination scenario is shown in Figure 2. The probability of an outbreak was reduced by increased vaccine uptake in the VZ [odds ratio (OR) $0.969,95 \%$ CI $0.966-0.972, P<0.001]$ but neither the size of the VZ nor the use of reactive vaccination had a significant effect on the likelihood of an outbreak (not shown). Incursions in September generated a significantly lower proportion of outbreaks than in May (OR 0.047 , 95\% CI $0 \cdot 032-0 \cdot 070$, $P<0 \cdot 001$ ) and July (OR 0.055, 95\% CI 0.036-0.081, $P<0 \cdot 001)$.

\section{Spatial and temporal dynamics}

The predicted spatial spread and time-course of BTV infection in the absence of vaccination is shown in Figure 3. Incursions on each date result in some spread of BTV with later incursions resulting in fewer affected farms across a smaller area of GB. All 


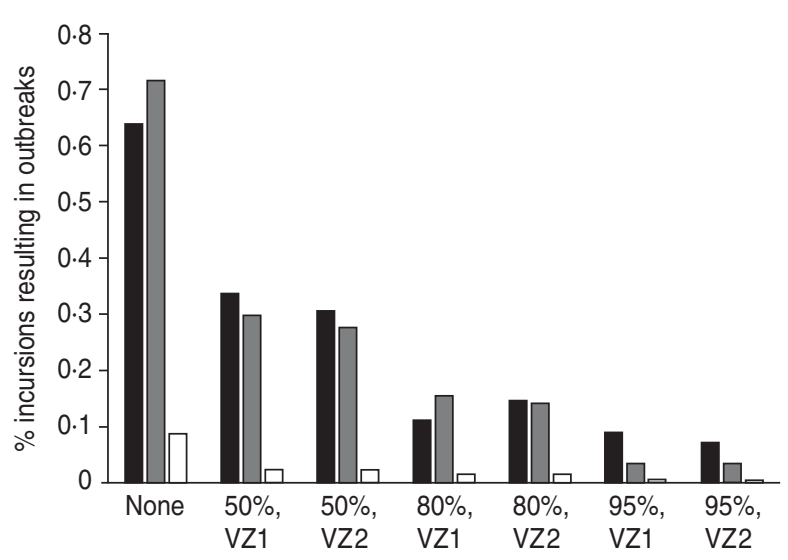

Fig. 2. Probability of an outbreak of bluetongue virus under different pre-emptive vaccination scenarios: $\mathbf{\square}$, May incursions; $\square$, July incursions; $\square$, September incursions. Labels indicate the vaccine uptake $(\%)$ and choice of zone. Results are based on the number of replicates of the model required to generate 30 outbreaks (defined as any spread from the initially infected farm).

vaccination strategies result in smaller outbreaks and less spatial spread (Figs 4 and 5; cf. Fig. 3). The impact of reactive vaccination is particularly evident in early incursions with larger radii resulting in smaller outbreaks (Fig. 4). Incursions into a vaccinated population, supplemented by a reactive strategy, result in smaller outbreaks than with a reactive strategy alone (Fig. 5; cf. Fig. $4 a, b$ ) with higher levels of uptake producing greater reductions in both the number of affected farms and the spatial spread (Fig. 5).

\section{Outbreak size}

The size of an outbreak (defined as total number of affected farms) under each vaccination scenario is shown in Figure $6 a$ for an incursion in May. Similar patterns were observed for simulated incursions in July (see Supplementary Fig. S1). Incursions in September (see Supplementary Fig. S2) produced significantly smaller outbreaks $(P<0 \cdot 001)$.

Increasing either the radius (coefficient in final GLM: $\quad b=0.021, \quad 95 \%$ CI -0.026 to -0.015 , $P<0.001)$ or uptake $(b=-0.005,95 \% \mathrm{CI}-0.009$ to $-0 \cdot 001, P<0 \cdot 001)$ for reactive vaccination reduced the size of an outbreak. There was also a significant interaction between the date of incursion and the radius for reactive vaccination; the later the incursion the smaller the reduction in outbreak size achieved by increasing the radius. Increased vaccine uptake in the VZ significantly reduced the size of an outbreak $(b=-0.009,95 \% \mathrm{CI}-0.013$ to $-0.005, P<0 \cdot 001)$ but the size of the zone was not significant $(P>0 \cdot 1)$ in determining the outbreak size.

\section{Morbidity, mortality and vaccine usage}

Morbidity (defined as total number of cattle and sheep infected) and mortality (defined as the total number of cattle and sheep dead) for each scenario are shown in Fig. 6b, $c$ for incursions in May (results for incursions in July and September can be found in Supplementary Figs S1 and S2). For early incursions (May or July) the lowest morbidity levels occur with widespread reactive vaccination $(80 \%$ or $95 \%$ uptake within $100 \mathrm{~km}$ of infected premises). These scenarios require similar numbers of vaccine doses to other reactive plans and fewer than pre-emptive strategies, although there is considerable variation in the number of doses used (Fig. 6d). For later incursions (September) pre-emptive and reactive vaccination strategies result in comparable levels of morbidity. Disease-induced mortality is reduced by all forms of vaccination especially for early incursions, and for all vaccination scenarios the mean number of dead animals in an outbreak was $<15$.

\section{Sensitivity analysis}

\section{Probability of an outbreak}

The shape of the kernel did not significantly affect the probability of an outbreak, nor did it affect the predicted impact of vaccination on the probability of an outbreak (i.e. both the radius for reactive vaccination and the size of the VZ had no significant effect for all kernels). Increased vaccine efficacy significantly reduces the probability of an outbreak when the incursion occurs into a vaccinated population ( $80 \%$ uptake in VZ 1), but not when only reactive vaccination is used.

\section{Outbreak size}

Outbreaks simulated using the exponential kernel are larger than those using the Gaussian kernel but the difference is not significant. However, both the fattailed and FMD kernels produced significantly larger outbreaks than either the Gaussian or exponential kernel (fat-tailed vs. Gaussian: $b=2 \cdot 74,95 \%$ CI 2.13-3.34, $\quad P<0 \cdot 0001$; fat-tailed vs. exponential: $b=2 \cdot 52,95 \%$ CI $1.91-3 \cdot 12, P<0.0001$; FMD vs. Gaussian: $b=2 \cdot 27,95 \%$ CI $1 \cdot 66-2 \cdot 87, P<0 \cdot 0001$; FMD vs. exponential: $b=2 \cdot 52,95 \%$ CI $1 \cdot 44-2 \cdot 65$, $P<0 \cdot 0001)$. 
(a)

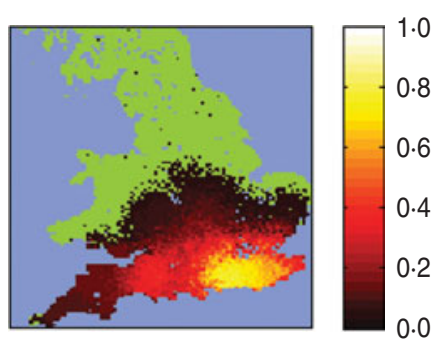

(c)

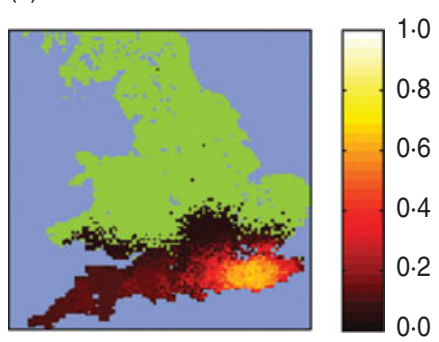

(e)

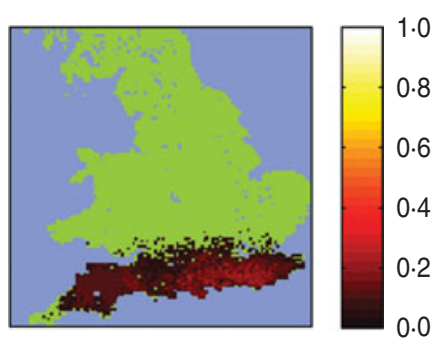

(b)

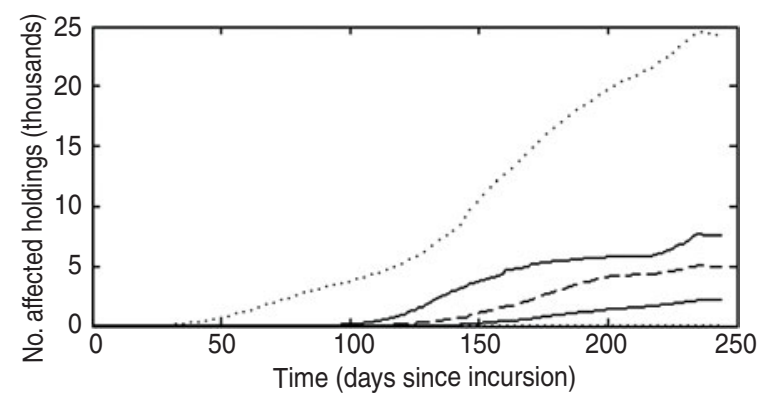

(d)

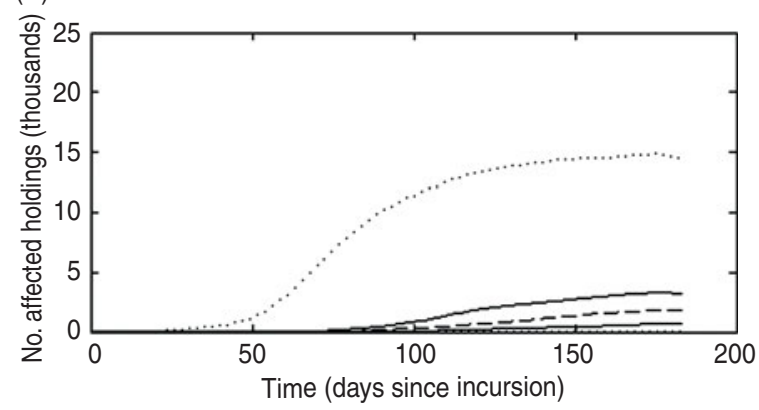

(f)

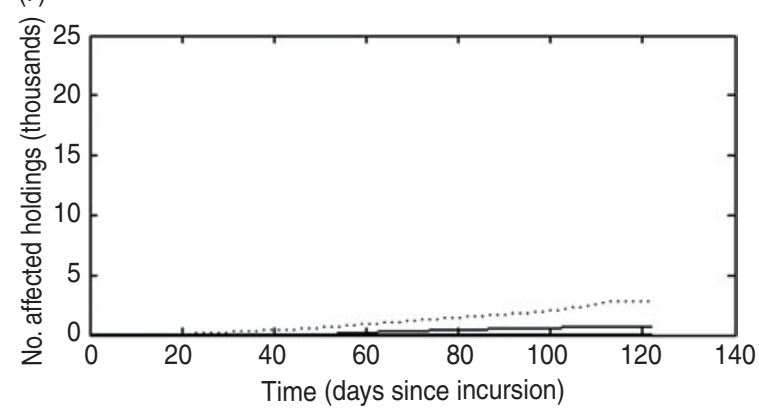

Fig. 3 [colour online]. Predicted spatial distribution and epidemic time-course of bluetongue virus serotype 1 (BTV-1) in Great Britain in the absence of vaccination for an incursion on 1 May $(a, b), 1$ July $(c, d)$ or 1 September $(e, f)$. The maps show the proportion of outbreaks (out of 30) for which at least one farm was affected by BTV-1 within each 5-km grid square. The time-courses show the number of affected farms on each day of the simulation:---, median; - , 25th and 75th percentiles; $\cdots \cdots, 2 \cdot 5$ th and $97 \cdot 5$ th percentiles of 30 outbreaks.

The predicted impact of reactive vaccination does not depend on the choice of kernel. As the radius is increased the size of an outbreak is significantly reduced for any kernel. Similarly, for any choice of kernel the declaration of a larger $\mathrm{VZ}$ produces smaller outbreaks but the effect is not significant.

The effect of reactive vaccination is sensitive to the level of vaccine efficacy. For low levels of vaccine efficacy $(50 \%, 60 \%, 70 \%)$ increasing the radius of the reactive $\mathrm{VZ}$ does not reduce the size of an outbreak. However, for higher efficacy larger radii produce significantly smaller outbreaks $(80 \%: b=-0 \cdot 011,95 \%$ CI -0.018 to $-0.0041, P<0.05 ; 90 \%: b=-0.012$, $95 \%$ CI -0.019 to $-0.0050, P<0.05 ; 100 \%: b=$ $-0.019,95 \%$ CI -0.026 to $-0.011, P<0.001)$. Increased vaccine efficacy does not significantly reduce the size of outbreaks for the pre-emptive vaccination strategy ( $80 \%$ uptake in VZ 1 with reactive vaccination uptake of $95 \%$ over a radius of $20 \mathrm{~km}$ ). This may be because insufficient farms are vaccinated for the increase in efficacy to have an effect, due to the relatively small size of the zone and the limited radius for reactive vaccination.

The results presented here are based on simulated incursions on 1 May. Similar patterns were found for incursions on 1 July.

\section{DISCUSSION}

Without vaccination simulations indicate that there is a high chance of an outbreak spreading beyond the initial site of infection (Fig. 2). The greatest risk is 
(a)

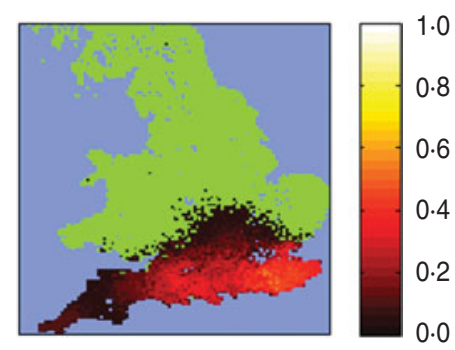

(c)

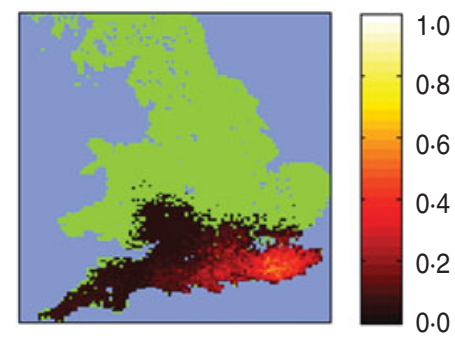

(e)

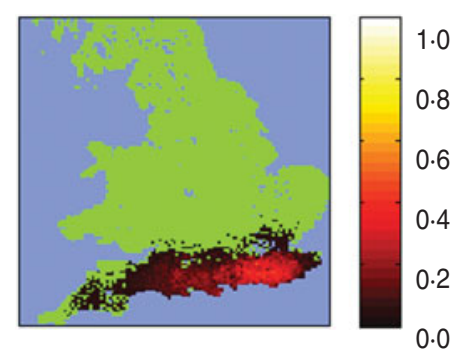

(b)

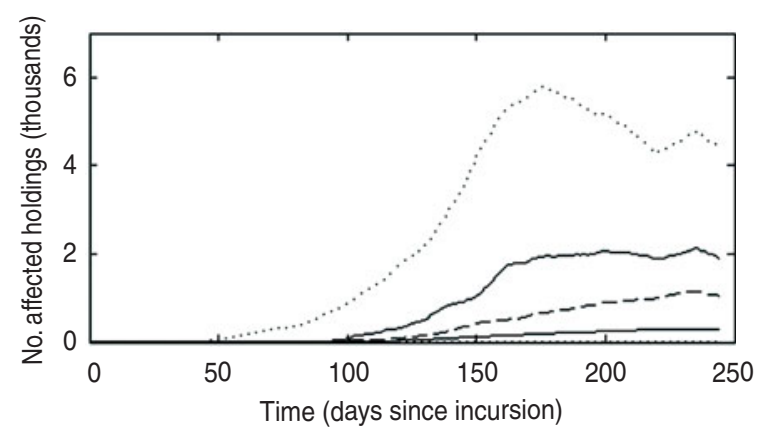

(d)

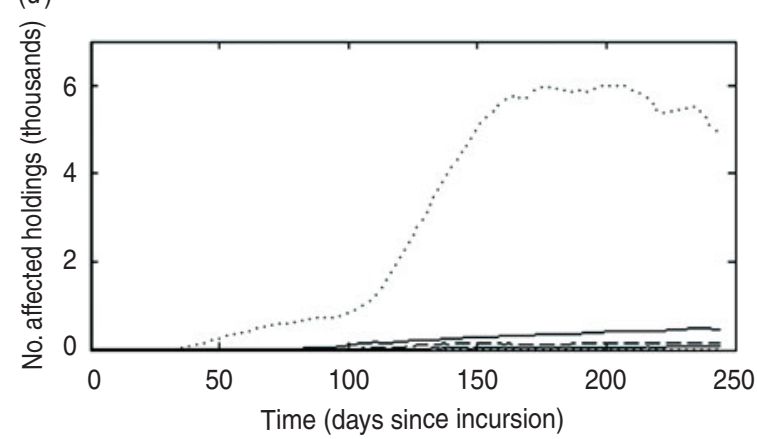

$(f)$

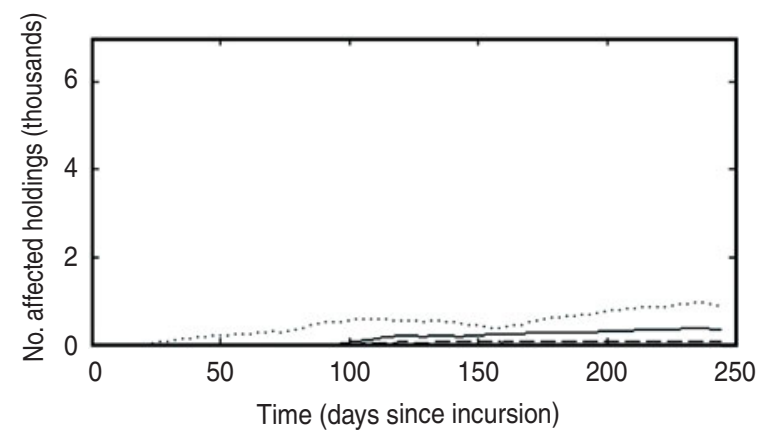

Fig. 4 [colour online]. Predicted spatial distribution and epidemic time-course of bluetongue virus serotype 1 (BTV-1) with reactive vaccination. Results presented are for incursions on 1 May with $95 \%$ reactive vaccine uptake within $20 \mathrm{~km}(a, b)$, $50 \mathrm{~km}(c, d)$ or $100 \mathrm{~km}(e, f)$ of infected premises. The maps show the proportion of outbreaks (out of 30) for which at least one farm was affected by BTV-1 within each $5-\mathrm{km}$ grid square. The time-courses show the number of affected farms on each day of the simulation:---, median,,- 25 th and 75 th percentiles; $\cdots, \cdot 2 \cdot 5$ th and $97 \cdot 5$ th percentiles of 30 outbreaks.

from an incursion earlier in the year (May, July). The earlier an outbreak occurs the greater the extent of the outbreak in terms of both its spatial spread and the number of farms affected, which is a consequence of the additional time available for BTV to spread. Furthermore, the predicted outbreak size for incursions to the south coast of England in September (Fig. $3 e, f$ ) are larger than those predicted by Szmaragd and co-workers [11] who simulated incursions to eastern England at the same time. This reflects the density of livestock, which is higher in the southern counties compared to East Anglia.
Reactive vaccination alone does not significantly affect the probability that an outbreak will take off because secondary spread will typically occur before disease is detected and vaccine can be deployed. When incursions occur into a vaccinated population the probability of an outbreak is significantly reduced compared to an unvaccinated population (Fig. 2). Higher levels of vaccine uptake in the VZ resulted in fewer outbreaks but changing the size of the zone (from VZ 1 to VZ 2) did not significantly impact the probability of the outbreak spreading, principally because incursions were assumed to occur only in VZ 1. 
(a)

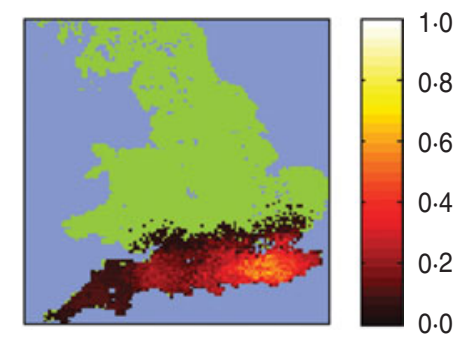

(c)

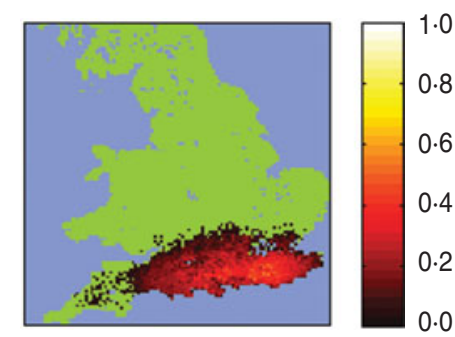

(e)

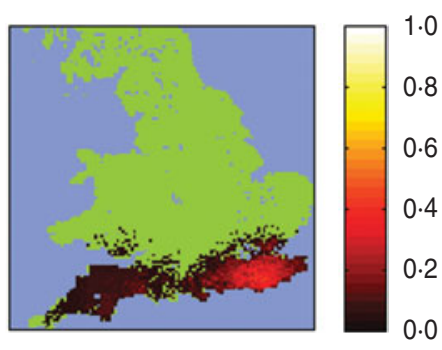

(b)

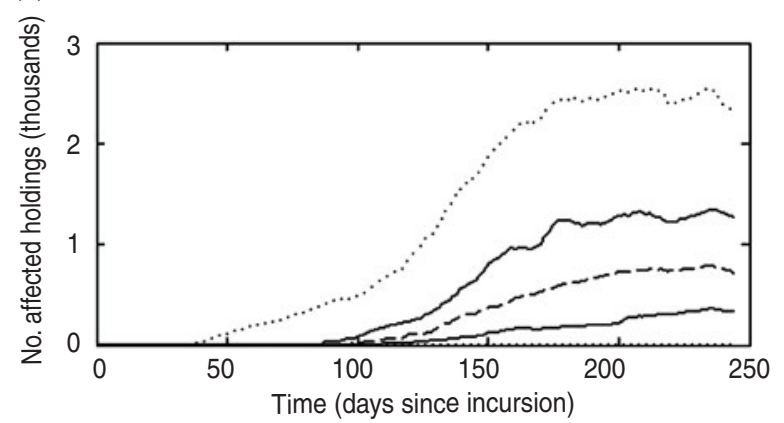

(d)
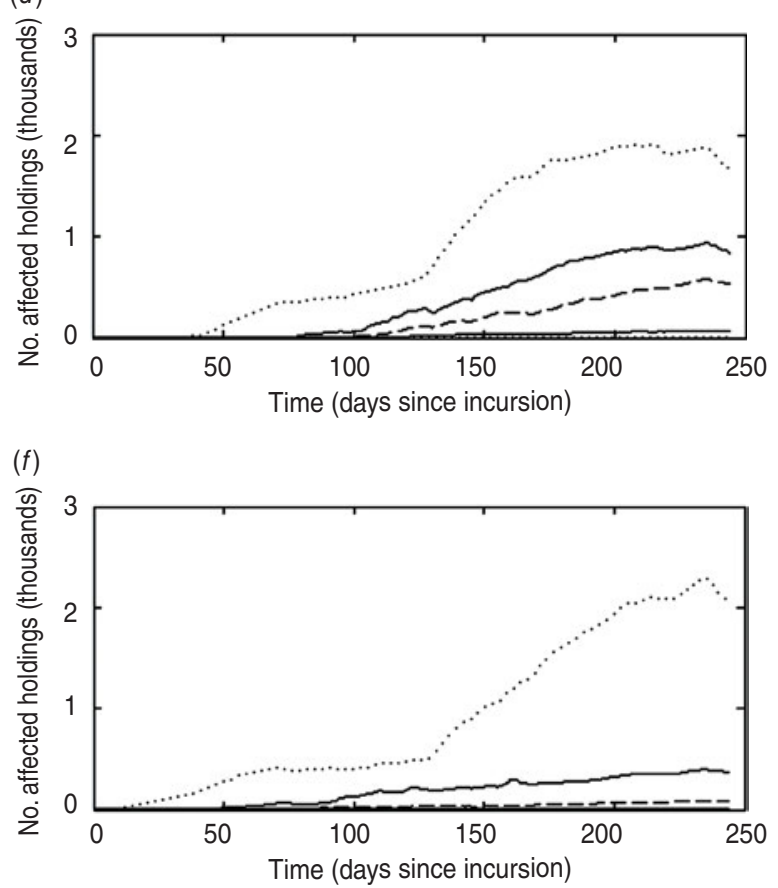

Fig. 5 [colour online]. Predicted spatial distribution and epidemic time-course of bluetongue virus serotype 1 (BTV-1) for incursions into a vaccinated population. Results presented are for incursions on 1 May with uptake of $50 \%(a, b), 80 \%(c, d)$ or $95 \%(e, f)$ in zone 2 and $95 \%$ reactive vaccine uptake within $20 \mathrm{~km}$ of infected premises. The maps show the proportion of outbreaks (out of 30) for which at least one farm was affected by BTV-1 within each 5-km grid square. The time-courses show the number of affected farms on each day of the simulation:---, median, - , 25th and 75 th percentiles; $\cdots \cdots, 2 \cdot 5$ th and 97.5th percentiles of 30 outbreaks.

Both reactive and pre-emptive vaccination reduced the spatial extent and number of affected premises when secondary spread occurs. Increasing the target area for reactive vaccination or the level of uptake both result in greater reductions in the size of the outbreak. For pre-emptive vaccination higher uptake produced smaller outbreaks but no significant effect was observed for the size of the zone. This is a consequence of assuming that, whichever zone is used for vaccination, the incursion will only occur into the area covered by VZ 1 . This suggests that provided the $\mathrm{VZ}$ covers the region at risk of an incursion there is little benefit in vaccinating over a wider area. Both forms of vaccination reduce the number of infected animals with the lowest levels of host morbidity achieved by large-scale reactive vaccination over a radius of $100 \mathrm{~km}$.

The impact of reactive vaccination is dependent on the timing of the incursion. For incursions which occur later in the year reactively vaccinating in response to the outbreak has less affect on the outbreak size because there is insufficient time for vaccine to be delivered and for livestock to reach full protection before transmission stops due to lower temperatures. This suggests that if an incursion occurs late in the year it may not be worth initiating a reactive 

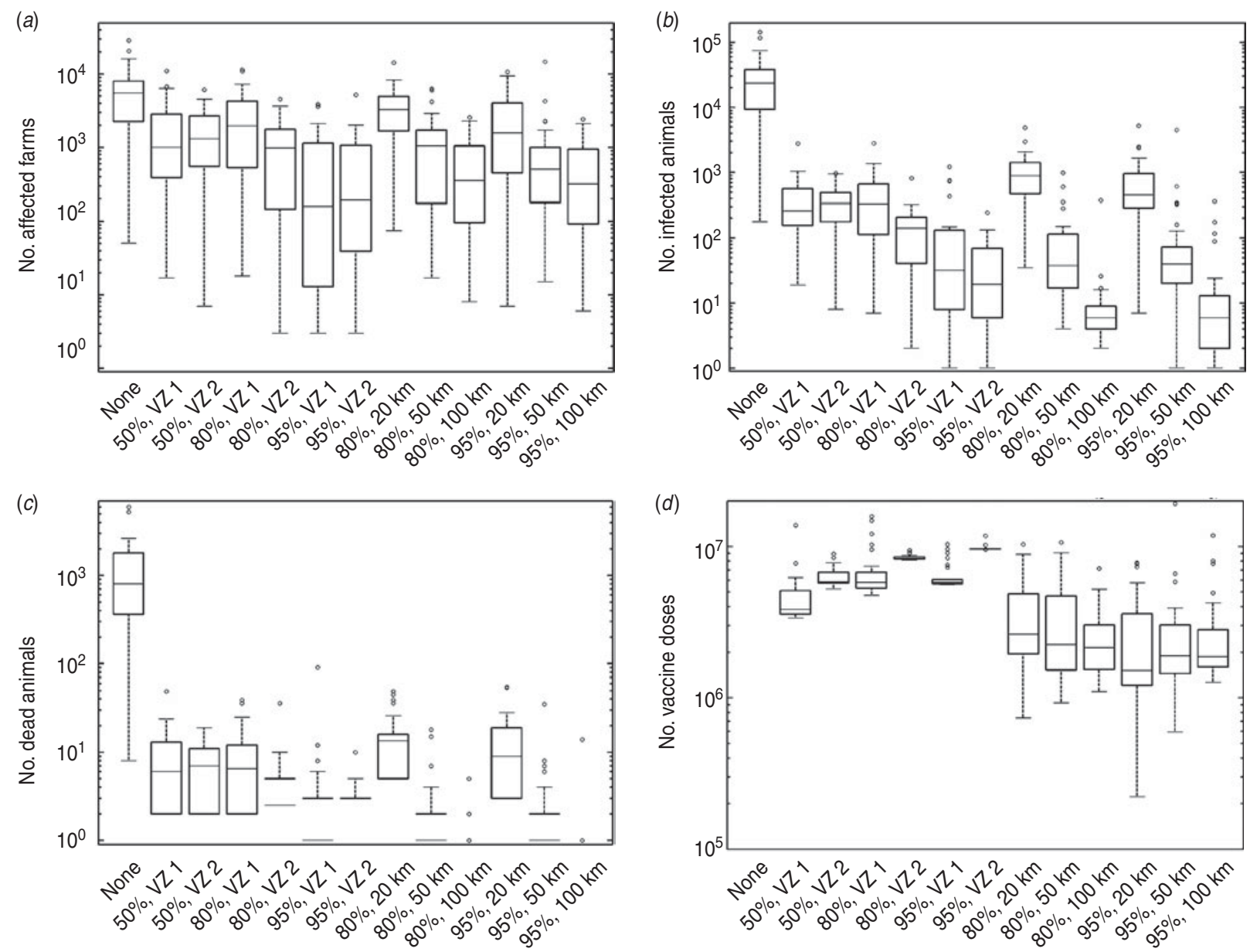

Fig. 6. Number of $(a)$ affected farms, $(b)$ infected animals, $(c)$ dead animals, $(d)$ vaccine doses used in different scenarios for incursions on 1 May. Labels indicate the vaccine uptake and (i) choice of zone or (ii) radius of reactive vaccination. Bars show the median, boxes show the lower and upper quartiles, whiskers show 1.5 times the interquartile range and open circles indicate outlying values. Results are based on 30 simulated outbreaks.

vaccination campaign. However, this study has only considered the short-term dynamics of the disease, from the date of incursion to the end of the year when transmission would be expected to have stopped. To assess the longer-term benefits of different vaccination strategies and, in particular, the ability to prevent the re-emergence of the disease in the following year, would require a greater understanding of the overwintering mechanisms of the virus [19].

Few studies have compared the impact of preemptive and reactive vaccination strategies against the same incursion, whether for BTV or for other livestock diseases. An earlier study of the impact of vaccination against BTV-8 in Scotland did consider both reactive and pre-emptive strategies [20]. Although that study found some evidence that preemptive vaccination had a larger effect than reactive vaccination, the authors did not directly compare reactive and pre-emptive strategies against the same incursion. Similarly, pre-emptive (or prophylactic) vaccination against FMD was shown to be more effective at controlling outbreaks (with higher levels of coverage resulting in smaller outbreaks) than reactive vaccination [21]. However, the reactive strategies assumed a long delay ( $>14$ days) between identification of an infected premises and the start of vaccination [21] and shortening this delay increases the effectiveness of a reactive strategy [22]. For reactive vaccination strategies the reduction in outbreak size with increasing radius has been demonstrated for a number of other livestock diseases including classical swine fever [23], highly pathogenic avian influenza [24] and FMD [22]. Furthermore, Tildesley et al. [22] showed that logistical constraints result in an optimal radius. Here we have assumed that logistical constraints are not limiting, which largely reflects the longer timescales over which vaccination takes place for BTV compared to FMD and because, in the case of BTV, it 
is implemented by farmers themselves and not special vaccination teams.

In addition to their ability to control the spread of disease it is also important to consider the economic costs of different vaccination strategies. Pre-emptive strategies carry the largest cost in terms of the purchase of vaccine and would also involve the costs of establishing the VZ, including losses incurred through restrictions placed on the movement of livestock out of the zone and the increased surveillance required to demonstrate disease-free status. While a purely reactive strategy would require less vaccine to be used and avoid the need for movement restrictions in the absence of disease it would involve the implementation of a surveillance scheme to ensure that vaccine was deployed effectively in the event of an outbreak.

In a recent study of the 2006 outbreak of BTV-8 in Belgium, Germany and The Netherlands, de Koeijer and co-workers [25] found that between-farm spread was best described by a kernel with more long-range transmission than the Gaussian kernel estimated by Szmaragd and co-workers [11] which was used in the simulations presented here. The shape of the kernel significantly affects the simulated disease dynamics and, therefore, it is important that careful consideration is given to alternative kernels when using models to predict the spread of BTV. However, based on the results of our sensitivity analysis which included kernels with longer-range transmission (i.e. the fattailed and FMD kernels, [11]), we anticipate that the relative benefits of different vaccination strategies are independent of the choice of kernel.

Vaccine efficacy influences the predicted effect of reactive vaccination on the spread of BTV. For low values of efficacy $(\leqslant 70 \%)$ increasing the spatial coverage of reactive vaccination does not significantly reduce the spread of disease, but at higher efficacies $(\geqslant 80 \%)$ increasing the spatial coverage of reactive vaccination does significantly reduce spread. A similar threshold efficacy (of between $70 \%$ and $80 \%$ ) has been identified for BTV previously [12], and reflects the basic reproduction number for this disease [26]. Moreover, the impact of efficacy on the success of a reactive vaccination campaign suggests that good estimates of vaccine efficacy are important in determining the best control strategy.

Previous analysis [12] has shown that model predictions are also sensitive to the temperature data used in the model, with 2006 (a warmer year) producing larger outbreaks than 2007 (a cooler year). However, changing the temperature should have no effect on the relative impact of the different vaccination strategies.

In the model the effects of movement restrictions on transmission between farms were ignored and, hence, the predictions represent a worst-case scenario for spread. Previous analysis of the 2007 BTV-8 outbreak in GB suggested that simulating movement restrictions by restricting spread to the protection zone had little impact on the model predictions with a Gaussian or exponential kernel, but they substantially reduced predicted spread for a fat-tailed or FMD kernel [11]. Consequently, including movement restrictions is unlikely to affect the predictions using either a Gaussian or exponential kernel and, indeed, we note that BTV is largely restricted to VZ 1 for these scenarios. For either the fat-tailed or FMD kernel, however, movement restrictions will reduce spread, although there will still be higher levels of spread compared to the Gaussian or exponential kernels. Moreover, there will be a greater risk of infection reaching the edges of the zone raising the possibility of spread (via vectors) to outside the zone. To fully assess the impacts of movement restrictions it is necessary to separate out the major routes of between-farm transmission (vector dispersal and livestock movements), and future development of BTV transmission models should focus on explicitly incorporating these different mechanisms (see e.g. [27]).

\section{CONCLUSIONS}

Both pre-emptive and reactive vaccination limited the spread of BTV in the event of an outbreak. However, only pre-emptive vaccination was found to reduce the probability of an outbreak following the initial introduction of BTV. Consequently, if the risk of introduction is high there is a stronger case for the use of pre-emptive vaccination. The timing of the incursion had a significant impact on the disease dynamics and the potential benefits of vaccination. In particular if BTV was introduced late in the year reactive vaccination was found to have little impact on the spread of disease and any decision to vaccinate reactively should take into consideration the natural impact of seasonality on disease transmission. The direct and indirect costs should be considered when selecting the best control strategy. While no formal economic analysis has been performed, reactive strategies used the fewest vaccine doses. However, indirect costs, in particular the impact on the livestock trade of 
declaring a PZ or VZ for the purposes of pre-emptive vaccination, must also be quantified.

\section{SUPPLEMENTARY MATERIAL}

For supplementary material accompanying this paper, visit http://dx.doi.org/10.1017/S0950268812000532.

\section{ACKNOWLEDGEMENTS}

This work was funded by the Department for Environment, Food and Rural Affairs (grant codes: SE4209 and SE4204).

\section{DECLARATION OF INTEREST}

None.

\section{REFERENCES}

1. Purse BV, et al. Climate change and the recent emergence of bluetongue in Europe. Nature Reviews Microbiology 2005; 3: 171-181.

2. Saegerman C, Berkvens D, Mellor PS. Bluetongue epidemiology in the European Union. Emerging Infectious Diseases 2008; 14: 539-544.

3. Wilson AJ, Mellor PS. Bluetongue in Europe: past, present and future. Philosophical Transactions of the Royal Society of London, Series B: Biological Sciences 2009; 364: 2669-2681.

4. Elbers AR, et al. Field observations during the Bluetongue serotype 8 epidemic in 2006. II. Morbidity and mortality rate, case fatality and clinical recovery in sheep and cattle in the Netherlands. Preventive Veterinary Medicine 2008; 87: 31-40.

5. Anon. Report on the distribution of bluetongue infection in Great Britain on 15 March 2008. London, UK: Department for Environment, Food and Rural Affairs, 2008.

6. Burgin L, Gloster J, Mellor PS. Why were there no outbreaks of bluetongue in the UK during 2008. Veterinary Record 2009; 164: 384-387.

7. Mintiens K, et al. Possible routes of introduction of bluetongue virus serotype 8 into epicentre of the 2006 epidemic in north-western Europe. Preventive Veterinary Medicine 2008; 87: 131-144.

8. Vandenbussche F, et al. Emergence of bluetongue serotypes in Europe, Part 1: Description and validation of four real-time RT-PCR assays for the serotyping of bluetongue viruses BTV-1, BTV-6, BTV-8 and BTV-11. Transboundary and Emerging Diseases 2009; 56: 346-354.

9. Gloster J, et al. Will bluetongue come on the wind to the United Kingdom in 2007? Veterinary Record 2007; 160: 422-426.
10. Gloster $\mathbf{J}$, et al. Assessing the risk of windborne spread of bluetongue in the 2006 outbreak of disease in northern Europe. Veterinary Record 2007; 160: 54-56.

11. Szmaragd C, et al. A modeling framework to describe the transmission of bluetongue virus within and between farms in Great Britain. PLoS ONE 2009; 4: e7741.

12. Szmaragd C, et al. The spread of bluetongue virus serotype 8 in Great Britain and its control by vaccination. PLoS ONE 2010; 5: e9353.

13. Jones AR, et al. The U.K. Met Office's next-generation atmospheric dispersion model, NAME III. In: Borrego C, Norman AL, eds. Air Pollution Modeling and its Application XVII (Proceedings of the 27th NATO/ CCMS International Technical Meeting on Air Pollution Modelling and its Application). New York: Springer, 2007, pp. 580-589.

14. Burgin L. The impacts of weather and climate change on the windborne spread of bluetongue into the United Kingdom (Ph.D. Thesis). University of Exeter, 2011.

15. Met Office. MIDAS land surface stations data (1853current) (http://badc.nerc.ac.uk/view/badc.nerc.ac.uk_ ATOM_dataent_ukmo-midas). Accessed September 2011.

16. Chis Ster I, Ferguson NM. Transmission parameters of the 2001 foot and mouth epidemic in Great Britain. PLoS ONE 2007; 2: e502.

17. Chis Ster I, Singh B, Ferguson N. Epidemiological inference for partially observed epidemics: the example of the 2001 foot and mouth epidemic in Great Britain. Epidemics 2009; 1: 21-24.

18. R Development Core Team. R: A Language and Environment for Statistical Computing. Vienna, Austria: R Foundation for Statistical Computing, 2011.

19. Wilson A, Darpel K, Mellor PS. Where does bluetongue virus sleep in the winter? PLoS Biology 2008; 6: e210.

20. Szmaragd C, Gunn GJ, Gubbins S. Assessing the consequences of an incursion of a vector-borne disease. II. Spread of bluetongue in Scotland and impact of vaccination. Epidemics 2010; 2: 139-147.

21. Keeling MJ, et al. Modelling vaccination strategies against foot-and-mouth disease. Nature 2003; 421: 136-142.

22. Tildesley MJ, et al. Optimal reactive vaccination strategies for a foot-and-mouth outbreak in the UK. Nature 2006; 440: 83-86.

23. Backer JA, et al. Modelling the effectiveness and risks of vaccination strategies to control classical swine fever epidemics. Journal of the Royal Society Interface 2009; 6: 849-861.

24. Truscott J, et al. Control of a highly pathogenic H5N1 avian influenza outbreak in the GB poultry flock. Proceedings of the Royal Society B: Biological Sciences 2007; 274: 2287-2295.

25. de Koeijer AA, et al. Quantitative analysis of transmission parameters for bluetongue virus serotype 8 in 
Western Europe in 2006. Veterinary Research 2011; 42: 53-61.

26. Gubbins S, et al. Assessing the risk of bluetongue to UK livestock: uncertainty and sensitivity analysis of a temperature-dependent model for the basic reproductive number. Journal of the Royal Society Interface 2008; 5: 363-371.

27. Ducheyne E, et al. A stochastic predictive model for the natural spread of bluetongue. Preventive Veterinary Medicine 2011 ; 99: 48-59. 